\title{
Are pediatric Open Access journals promoting good publication practice? An analysis of author instructions
}

Joerg J Meerpohl ${ }^{1,2^{*}+}$, Robert F Wolff ${ }^{1,3 \dagger}$, Gerd Antes ${ }^{1}$ and Erik von Elm ${ }^{1,4}$

\begin{abstract}
Background: Several studies analyzed whether conventional journals in general medicine or specialties such as pediatrics endorse recommendations aiming to improve publication practice. Despite evidence showing benefits of these recommendations, the proportion of endorsing journals has been moderate to low and varied considerably for different recommendations. About half of pediatric journals indexed in the Journal Citation Report referred to the Uniform Requirements for Manuscripts of the International Committee of Medical Journal Editors (ICMJE) but only about a quarter recommended registration of trials. We aimed to investigate to what extent pediatric openaccess (OA) journals endorse these recommendations. We hypothesized that a high proportion of these journals have adopted recommendations on good publication practice since OA electronic publishing has been associated with a number of editorial innovations aiming at improved access and transparency.

Methods: We identified 41 journals publishing original research in the subject category "Health Sciences, Medicine (General), Pediatrics" of the Directory of Open Access Journals http://www.doaj.org. From the journals' online author instructions we extracted information regarding endorsement of four domains of editorial policy: the Uniform Requirements for Manuscripts, trial registration, disclosure of conflicts of interest and five major reporting guidelines such as the CONSORT (Consolidated Standards of Reporting Trials) statement. Two investigators collected data independently.
\end{abstract}

Results: The Uniform Requirements were mentioned by 27 (66\%) pediatric OA journals. Thirteen (32\%) required or recommended trial registration prior to publication of a trial report. Conflict of interest policies were stated by 25 journals (61\%). Advice about reporting guidelines was less frequent: CONSORT was referred to by 12 journals (29\%) followed by other reporting guidelines (MOOSE, PRISMA or STARD) (8 journals, 20\%) and STROBE (3 journals, 7\%). The EQUATOR network, a platform of several guideline initiatives, was acknowledged by 4 journals (10\%). Journals published by OA publishing houses gave more guidance than journals published by professional societies or other publishers.

Conclusions: Pediatric OA journals mentioned certain recommendations such as the Uniform Requirements or trial registration more frequently than conventional journals; however, endorsement is still only moderate. Further research should confirm these exploratory findings in other medical fields and should clarify what the motivations and barriers are in implementing such policies.

\footnotetext{
* Correspondence: meerpohl@cochrane.de

† Contributed equally

${ }^{1}$ German Cochrane Center, Institute of Medical Biometry and Medical Informatics, University Medical Center Freiburg, Berliner Allee 29, D-79110

Freiburg, Germany

Full list of author information is available at the end of the article
}

\section{() Biomed Central}

(c) 2011 Meerpohl et al; licensee BioMed Central Ltd. This is an Open Access article distributed under the terms of the Creative Commons Attribution License (http://creativecommons.org/licenses/by/2.0), which permits unrestricted use, distribution, and reproduction in any medium, provided the original work is properly cited. 


\section{Background}

Medical research reports and publication practice in biomedicine have been under increased scrutiny over the last decades. Selective reporting of study results and related publication bias has been confirmed in several empirical studies in different disciplines and settings [1]. Further, there is continued concern regarding conflicts of interest that are not disclosed by article authors [2] and cases of scientific misconduct [3]. Non-reporting of study results has been identified also in pediatric research [4]. For instance, significant differences in the risk-benefit profile of selective serotonin re-uptake inhibitors (SSRIs) in children were found in a meta-analysis of studies published in peer-reviewed journals and unpublished data [5]. The data suggesting that SSRIs are linked to an increased risk of suicide or suicidal thoughts had not been published [6].

In the past, several recommendations have been proposed to improve the reporting and publication practice in biomedicine: First, the International Committee of Medical Journal Editors (ICMJE) published the "Uniform Requirements for Manuscripts submitted to Biomedical Journals" [7]. This widely used guideline is currently endorsed by over 700 journals and covers issues such as ethical conduct and reporting of biomedical research, preparation and publishing of manuscripts and editorial policies. Second, the problem of publication bias and selective outcome reporting has been widely analyzed over the last 15 years [1]. Registration of clinical trials and studies of other types prior to patient enrollment has been advocated as an important first step to tackle this problem. Third, authors but also journal editors and reviewers might have financial ties or personal interests in conflict with an article being submitted for publication [8,9]. The debate about this problem has led journals to require disclosure of potential conflicts of interest. Recently, a uniform conflict of interest disclosure form was proposed jointly by major medical journals [10]. Finally, published reporting guidelines such as the CONSORT Statement provide guidance to authors and aim at improving the completeness and accuracy of publications $[11,12]$. Further, they facilitate the critical appraisal by readers. Endorsement and implementation of these reporting guidelines has been studied for general medicine journals $[13,14]$ but less so for journals in specialties such as pediatrics.

Journals and their editors play a key role in promoting and ensuring transparency in biomedical publishing. Previously, we focused on pediatric journals indexed in the Journal Citation Report and found that the advice given to authors regarding the above mentioned four domains was moderate to low [15]. Reflecting these findings, we wondered whether the low uptake might be due to hesitation or even reluctance of editors of these journals to experiment with editorial procedures.

Open-access electronic publishing has been associated with a number of editorial innovations aiming at improved access to and transparency of research results $[16,17]$. The new model was a response to the dilemma between increasing prices for journal subscriptions on one side and decreasing resources of academic institutions to finance access to the scientific literature. Open Access publications are generally made available online to anyone anywhere with no charges for access while recovering costs by charging publication fees from authors. Open Access journals usually provide peer review like journals following the conventional publishing model. It has been argued that electronic Open Access publishing does not change significantly content and quality of research articles but improves access to research findings [18].

We wondered whether journals adopting this new publication model take up recommendations which aim to ensure publication of research results in an unbiased and transparent manner. We therefore set out to elucidate the coverage of the four domains Uniform Requirements, trial registration, conflicts of interest and reporting guidelines in Open Access pediatric journals. We then compared our results with findings from "conventional" JCR-indexed pediatric journals analyzed earlier [15].

\section{Methods}

We accessed the Directory of Open Access Journals http://www.doaj.org on $4^{\text {th }}$ of September 2009, identified 43 journals listed in the "Health Sciences - Medicine (General) - Pediatrics" category and extracted information on their start year and publication language. We excluded one journal that does not publish original research articles (Foro Pediátrico) and another journal (Pediatric Cardiology Today) because it was continued as one of the included journals (Congenital Cardiology Today) in 2005. From the websites of the 41 included journals we downloaded the author instructions in September 2009. Two authors (JJM and RFW) read each document and classified information about the geographical location of the main editorial office using the following groups: Africa, Australasia, Europe (without UK), United Kingdom (UK), North America, and South America. Further, we defined three categories of publishers: open access publishing houses, professional societies/academic institutions and other publishers. Using relevant keywords in electronic full text searches we then identified any information on the following four domains of good publication practice: 
- Endorsement of the ICMJE Uniform Requirements, [7]

- Requirement of trial registration,

- Editorial policies for disclosure of conflicts of interests,

- Endorsement of five reporting guidelines and related explanatory papers:

- CONSORT (Consolidated Standards of Reporting Trials) $[19,20]$ and its extensions;

- STROBE (Strengthening the Reporting of Observational Studies in Epidemiology); [21,22]

- STARD (Standards for Reporting of Diagnostic Accuracy); [23,24]

- MOOSE (Meta-analysis of Observational Studies in Epidemiology); [25]

- QUOROM (Quality of Reporting of Meta-analyses) [26] which has recently been revised and renamed to PRISMA (Preferred Reporting Items for Systematic Reviews and Meta-Analyses) $[27,28]$.

For each of these items, we analyzed the wording of the author instructions and determined whether it was "required" (i.e. a submitted manuscript would not be accepted if the item was not considered) or "recommended" (i.e. its use or fulfillment ought to be considered). Two investigators (JJM, RFW) extracted and categorized information independently. Discrepancies occurred in less than $1 \%$ of items and were all resolved by discussion among the investigators.

For comparison, data from a previous study on 69 pediatric journals indexed in the Journal Citation Report (JCR) were used [15].

\section{Results}

The editorial offices of the 41 included journals were located in 21 countries all over the world. Eleven were located in Australasia; fourteen in Europe, nine in South America, six in North America and one in Africa (Table 1). These 41 journals were published by 32 different publishers. Thirteen journals were published by open access publishing houses: BioMed Central $(\mathrm{n}=5)$, Medknow Publications $(\mathrm{n}=4)$, Hindawi Publishing Corporation $(\mathrm{n}=$ $2)$, Libertas Academica $(n=1)$ and Bentham $(n=1)$. Fourteen were published either by regional/national professional organisations or academic institutions, and another fourteen by various other publishers. One journal (Indian Pediatrics) was indexed in both the Directory of Open Access Journals and the Journal Citation Report 2008.

Only one journal, "Revista Chilena de Pediatria", started publishing in the 1980s. Six journals started in the 1990 s, while most of them $(n=34)$ started publishing in 2000 or later. The majority are published in English $(\mathrm{n}=23)$, while 6 are published in Spanish, 4 in Turkish and 2 in Portuguese. Six journals publish their articles in two or more different languages with all but one publishing in English.

\section{ICMJE Uniform requirements}

The ICMJE Uniform Requirements for Manuscripts were mentioned by 27 journals (66\%) (Table 1). Of those, 23 (85\%) referred to the web address http://www.icmje.org, where the full document can be downloaded. The Uniform Requirements were most often mentioned in one of the following contexts: (1) the journals support and follow the Uniform Requirements in general, (2) further information on trial registration can be found in the Uniform Requirements and (3) they are recommended as a reference document for manuscript style (e.g. formatting of bibliographies).

\section{Trial registration}

Trial registration was mentioned in thirteen of the 41 journals $(32 \%)$, out of which nine required and four recommended trial registration prior to publication of a manuscript (Table 1). Several of these journals referred to the ICMJE website for further guidance on trial registration. Two journals did not mention any suitable trial registry in their author instructions.

\section{Conflict of interest policies}

Policies for disclosure of conflicts of interest were found in the author instructions of 26 journals (63\%) (Table 1). Six journals stated that they publish information on potential conflicts together with the manuscript. For twenty journals, the author instructions did not specify how the authors' conflicts of interests are handled. The remaining fifteen journals did not provide any information on disclosure of potential conflicts of authors.

\section{Reporting guidelines}

The CONSORT statement was the reporting guideline that was cited most often, i.e. in 12 of 41 journals, 29\% (Table 1). Eight journals required authors to follow the CONSORT checklist when preparing manuscripts reporting on trials or to submit a completed checklist together with the manuscript. Four recommended the use of the CONSORT statement when preparing manuscripts. The web address, http://www.consort-statement.org, was given by eleven journals. Each of the reporting guidelines STARD, MOOSE and QUOROM/PRISMA was mentioned in the author instructions of eight journals (20\%). The STROBE statement was mentioned by three journals (7\%). The EQUATOR network http://www.equator-network.org, an initiative collating several reporting guideline, was mentioned by four journals (10\%). 
Table 1 Recommendations provided in author instructions of Open Access (OA) pediatric journals

Recommendation Geographical location of editorial office No. (\% of column total)

\begin{tabular}{|c|c|c|c|c|c|c|c|c|c|c|}
\hline \multirow[t]{3}{*}{ Recommendation } & & \multicolumn{6}{|c|}{ Geographical location of editorial office No. (\% of column total) } & \multicolumn{3}{|c|}{ Category of publisher No. (\% of column total) } \\
\hline & \multirow{2}{*}{$\begin{array}{c}\text { All } \\
\text { journals } \\
N=41\end{array}$} & \multirow{2}{*}{$\begin{array}{l}\text { Africa } \\
\mathrm{N}=1\end{array}$} & \multirow{2}{*}{$\begin{array}{c}\text { Australasia } \\
\mathrm{N}=11\end{array}$} & \multicolumn{2}{|l|}{ Europe } & \multirow{2}{*}{$\begin{array}{c}\text { South } \\
\text { America } \\
\mathrm{N}=9\end{array}$} & \multirow{2}{*}{$\begin{array}{c}\text { North } \\
\text { America } \\
\mathrm{N}=6\end{array}$} & \multirow{2}{*}{$\begin{array}{c}\text { OA Publishing } \\
\text { House } \\
\mathrm{N}=13\end{array}$} & \multirow{2}{*}{$\begin{array}{c}\text { Other } \\
\text { publisher } \\
\mathrm{N}=14\end{array}$} & \multirow{2}{*}{$\begin{array}{c}\text { Professional } \\
\text { organization } \\
N=14\end{array}$} \\
\hline & & & & without UK $\mathrm{N}=11$ & UK $N=3$ & & & & & \\
\hline $\begin{array}{l}\text { ICMJE Uniform Requirements for } \\
\text { Manuscripts }\end{array}$ & $27(65.9)$ & $1(100)$ & $7(63.6)$ & $7(63.6)$ & $3(100)$ & $7(77.8)$ & $2(33.3)$ & $10(77.0)$ & $8(57.1)$ & $9(66.3)$ \\
\hline Trial registration & $13(31.7)$ & $1(100)$ & $3(27.3)$ & $2(18.2)$ & $3(100)$ & $3(33.3)$ & $1(16.7)$ & $8(61.5)$ & $1(7.1)$ & $4(28.6)$ \\
\hline Conflict of interest & $26(63.4)$ & $1(100)$ & $7(63.6)$ & $6(54.5)$ & $3(100)$ & $6(66.7)$ & $3(50)$ & $13(100)$ & $6(42.9)$ & $7(50.0)$ \\
\hline CONSORT & $12(29.3)$ & $1(100)$ & $5(45.5)$ & $1(9.1)$ & $3(100)$ & $1(11.1)$ & $1(16.7)$ & $9(69.2)$ & $0(0)$ & $3(21.4)$ \\
\hline STROBE & $3(7.3)$ & $1(100)$ & $2(18.2)$ & $0(0)$ & $0(0)$ & $0(0)$ & $0(0)$ & $2(15.4)$ & $0(0)$ & $1(7.1)$ \\
\hline STARD & $8(19.5)$ & $1(100)$ & $3(27.3)$ & $1(9.1)$ & $3(100)$ & $0(0)$ & $0(0)$ & $7(53.8)$ & $0(0)$ & $1(7.1)$ \\
\hline MOOSE & $8(19.5)$ & $1(100)$ & $3(27.3)$ & $1(9.1)$ & $3(100)$ & $0(0)$ & $0(0)$ & $7(53.8)$ & $0(0)$ & $1(7.1)$ \\
\hline PRISMA (QUOROM) & $8(19.5)$ & $1(100)$ & $3(27.3)$ & $1(9.1)$ & $3(100)$ & $0(0)$ & $0(0)$ & $7(53.8)$ & $0(0)$ & $1(7.1)$ \\
\hline EQUATOR & $4(9.8)$ & $0(0)$ & $1(9.1)$ & $0(0)$ & $3(100)$ & $0(0)$ & $0(0)$ & $4(30.8)$ & $0(0)$ & $0(0)$ \\
\hline
\end{tabular}

ICMJE - International Committee of Medical Journal Editors, CONSORT - Consolidated Standards of Reporting Trials, STROBE - Strengthening the Reporting of Observational Studies in Epidemiology, STARD Standards of Reporting Diagnostic Accuracy, MOOSE - Meta-analysis of observational studies in epidemiology, PRISMA - Preferred Reporting Items for Systematic Reviews and Meta-Analyses, EQUATOR - Enhancing 


\section{Analysis according to geographical location and category of publisher}

First, the analysis according to geographical location of editorial office showed some variation in the guidance given: the three journals with an editorial office in the UK gave the most guidance while journals based in North America provided much less guidance (Table 1).

Second, we wondered whether the type of publisher influenced the amount of guidance given to authors and therefore analysed our findings according to category of publisher. The group of journals published by Open Access publishers $(n=13)$ offered the most guidance to their authors: the Uniform Requirements were mentioned by ten journals $(77 \%)$, while trial registration was required or recommended by eight (62\%). All thirteen journals described a conflict of interest policy on their website. The CONSORT statement was mentioned by nine journals (69\%), while STARD, MOOSE and PRISMA were referred to by seven journals (54\%). The EQUATOR network was mentioned by four journals (31\%) (Table 1). Recommendations regarding the four domains of good publication practice were less often mentioned by journals published by professional societies or academic institutions and by other publishers.

\section{Discussion}

We analyzed to what extent author instructions of Open Access pediatric journals reflect recommendations on four domains of editorial policy: Uniform Requirements, trial registration, conflicts of interest and reporting guidelines. The uptake of recommendations regarding these domains was moderate and varied considerably across journals. The proportion of Open Access journals giving advice in these four domains was slightly higher as compared to 69 journals listed in the Journal Citation Report 2008, except for conflict of interest policies (Table 2) [15].

The interpretation of these data and the comparison with our previous survey of author instructions [15] need to consider several aspects. First, the number of journals examined was not very large with 41 Open Access journals and 69 journals indexed in the Journal Citation Report. However, both selections did not represent samples drawn from a larger group but an analysis of all journals meeting the entry criteria. Second, clustering of journals that are run by the same publisher might have influenced our results. We aimed to address this by analysing the data by type of publisher. Third, our analysis was based on the policies as documented in the online instructions that potential authors would consult as first reference. We did not determine whether the editorial staff of the journals applies additional procedures that are not reflected in the author instructions e.g. to encourage or even enforce adherence of authors to the promoted policies. Such additional procedures could only be
Table 2 Recommendations provided in author instructions of 69 pediatric journals indexed in the Journal Citation Report [15]

\begin{tabular}{cc}
\hline Recommendation & $\begin{array}{c}\text { No. of journals giving } \\
\text { recommendation (\%) ( } \mathbf{~ = 6 9 )}\end{array}$ \\
\hline $\begin{array}{c}\text { ICMJE Uniform Requirements } \\
\text { for Manuscripts }\end{array}$ & $38(55)$ \\
\hline Trial registration & $16(23)$ \\
\hline Conflict of interest & $54(78)$ \\
\hline CONSORT & $14(20)$ \\
\hline STROBE & $3(4)$ \\
\hline STARD & $4(6)$ \\
\hline MOOSE & $3(4)$ \\
\hline PRISMA (QUOROM) & $4(6)$
\end{tabular}

ICMJE - International Committee of Medical Journal Editors, CONSORT Consolidated Standards of Reporting Trials, STROBE - Strengthening the Reporting of Observational Studies in Epidemiology, STARD - Standards of Reporting Diagnostic Accuracy, MOOSE - Meta-analysis of observational studies in epidemiology, PRISMA - Preferred Reporting Items for Systematic Reviews and Meta-Analyses.

identified by surveying editorial staff to elucidate their motivations to implement new policies or any barriers to do so. However, journal procedures were not the interest of our current study. Finally, our study is of exploratory nature. We are not aware of any other studies on author instructions of Open Access journals or comparisons with journals with another publishing model. One might speculate that the situation is similar in journals in general medicine or other specialties. Additional studies including other than pediatric journals should be undertaken to determine whether our results can be generalized to other Open Access journals.

Several of the included Open Access journals are more recent than the journals that are indexed in the Journal Citation Report. When they set up their author instructions they might have sought guidance on the most recent developments in good publication practice and consequently included recommendations on the four domains investigated in this survey from the start.

Although the empirical evidence on improvement of publication practice due to endorsement of these recommendations is still limited, providing such recommendations in the author instructions might be considered a means to enhance reporting quality by newly founded or less well-established journals [16,29].

Several of the included journals were run by publishers dedicated to Open Access publishing. This group of journals offered the most extensive guidance to their authors. It is conceivable that these publishers provided a master copy of author instructions to be adapted by the journal's editorial team. This circumstance might have contributed to the higher proportion of guidance that is offered by these OA journals. 
Finally, our analysis of author guidelines could be complemented by an assessment of the content published by the Open Access journals. Previous empirical studies have looked at the quality of published articles, in particular before and after introduction of reporting guidelines, but did not focus on different publishing models $[11,30]$.

\section{Conclusions}

Pediatric OA journals are a heterogeneous group of journals ranging from journals run by national societies to new publications launched by dedicated OA publishers. Overall, they do give at least as much guidance to authors as do conventional pediatric journals. Interestingly, differences exist between types of publishers. However, the uptake of good publication practices could still be improved in all groups. Whether these results can be generalized to Open Access journals in other specialties or in general medicine needs to be investigated. Reasons for differences should be elucidated e.g. by surveys of journals editors.

\section{Acknowledgements}

Christine Scheufele and Julia Lindenberg helped with locating and saving the respective websites and with data extraction.

We received no funding for this study. The open access publication of this work was supported by the Deutsche Forschungsgemeinschaft.

\section{Author details}

${ }^{1}$ German Cochrane Center, Institute of Medical Biometry and Medical Informatics, University Medical Center Freiburg, Berliner Allee 29, D-79110 Freiburg, Germany. ${ }^{2}$ Division of Pediatric Hematology \& Oncology, Department of Pediatrics, University Medical Center Freiburg, Mathildenstrasse 1, D-79106 Freiburg, Germany. ${ }^{3}$ Kleijnen Systematic Reviews Ltd, Unit 6, Escrick Business Park, Riccall Road, Escrick, York, YO19 6FD, UK. ${ }^{4}$ Swiss Paraplegic Research, Guido-A-Zaech-Strasse 4, CH-6207 Nottwil, Switzerland.

\section{Authors' contributions}

JJM and RFW conceived the study, developed the data extraction form, extracted the data and performed the data analysis. They also drafted the manuscript. GA helped with coordination of this project and critically discussed the manuscript. EvE was involved with data analysis, interpretation and provided statistical advice. He also was involved in writing the manuscript. All authors read and approved the final manuscript.

\section{Competing interests}

Erik von Elm is one of the authors of the STROBE Statement and Academic Editor of PLOS ONE, an Open Access journal not included in this study. The other authors are not aware of any potential conflicts of interest.

Received: 7 October 2010 Accepted: 9 April 2011 Published: 9 April 2011

\section{References}

1. Dwan K, Altman DG, Arnaiz JA, Bloom J, Chan AW, Cronin E, Decullier E, Easterbrook PJ, Von Elm E, Gamble C, et al: Systematic review of the empirical evidence of study publication bias and outcome reporting bias. PLOS ONE 2008, 3(8):e3081.

2. Bekelman JE, Li Y, Gross CP: Scope and impact of financial conflicts of interest in biomedical research: a systematic review. JAMA 2003, 289(4):454-465.

3. Titus SL, Wells JA, Rhoades LJ: Repairing research integrity. Nature 2008, 453(7198):980-982
4. Hetherington J, Dickersin K, Chalmers I, Meinert CL: Retrospective and prospective identification of unpublished controlled trials: lessons from a survey of obstetricians and pediatricians. Pediatrics 1989, 84(2):374-380

5. Whittington CJ, Kendall T, Fonagy P, Cottrell D, Cotgrove A, Boddington E: Selective serotonin reuptake inhibitors in childhood depression: systematic review of published versus unpublished data. Lancet 2004, 363(9418):1341-1345

6. Kondro W, Sibbald B: Drug company experts advised staff to withhold data about SSRI use in children. CMAJ 2004, 170(5):783.

7. International Committee of Medical Journal Editors: Uniform Requirements for Manuscripts Submitted to Biomedical Journals: Writing and Editing for Biomedical Publication. 2008 [http://www.icmje.org].

8. Smith R: Beyond conflict of interest. Transparency is the key. BMJ 1998, 317(7154):291-292.

9. Bhargava N, Qureshi J, Vakil N: Funding source and conflict of interest disclosures by authors and editors in gastroenterology specialty journals. Am J Gastroenterol 2007, 102(6):1146-1150.

10. Drazen JM, de Leeuw PW, Laine C, Mulrow C, Deangelis CD, Frizelle FA, Godlee F, Haug C, Hebert PC, Horton R, et al: Towards more uniform conflict disclosures: the updated ICMJE conflict of interest reporting form. BMJ 2010, 340:c3239.

11. Plint AC, Moher D, Morrison A, Schulz K, Altman DG, Hill C, Gaboury I: Does the CONSORT checklist improve the quality of reports of randomised controlled trials? A systematic review. Med J Aust 2006, 185(5):263-267.

12. Antes G: The new CONSORT statement. BMJ 2010, 340:c1432.

13. Altman DG: Endorsement of the CONSORT statement by high impact medical journals: survey of instructions for authors. BMJ 2005, 330(7499):1056-1057.

14. Hopewell S, Altman DG, Moher D, Schulz KF: Endorsement of the CONSORT Statement by high impact factor medical journals: a survey of journal editors and journal 'Instructions to Authors'. Trials 2008, 9(1):20.

15. Meerpohl JJ, Wolff RF, Niemeyer CM, Antes G, von Elm E: Editorial policies of pediatric journals: survey of instructions for authors. Arch Pediatr Adolesc Med 2010, 164(3):268-272.

16. Albert KM: Open access: implications for scholarly publishing and medical libraries. J Med Libr Assoc 2006, 94(3):253-262.

17. Bjork BC, Welling P, Laakso M, Majlender P, Hedlund T, Gudnason G: Open access to the scientific journal literature: situation 2009. PLOS ONE 2010, 5(6):e11273

18. Mackenzie Owen J: The Scientific Article in the Age of Digitization. Springer, Netherlands; 2007.

19. Schulz KF, Altman DG, Moher D: CONSORT 2010 statement: updated guidelines for reporting parallel group randomised trials. PLOS Med 2010, 7(3):e1000251.

20. Moher D, Hopewell S, Schulz KF, Montori V, Gotzsche PC, Devereaux PJ, Elbourne D, Egger M, Altman DG: CONSORT 2010 Explanation and Elaboration: Updated guidelines for reporting parallel group randomised trials. J Clin Epidemiol 2010, 63(8):e1-37.

21. von Elm E, Altman DG, Egger M, Pocock SJ, Gotzsche PC Vandenbroucke JP: The Strengthening the Reporting of Observational Studies in Epidemiology (STROBE) statement: guidelines for reporting observational studies. Ann Intern Med 2007, 147(8):573-577.

22. Vandenbroucke JP, von Elm E, Altman DG, Gotzsche PC, Mulrow CD, Pocock SJ, Poole C, Schlesselman JJ, Egger M: Strengthening the Reporting of Observational Studies in Epidemiology (STROBE): explanation and elaboration. Ann Intern Med 2007, 147(8):W163-194.

23. Bossuyt PM, Reitsma JB, Bruns DE, Gatsonis CA, Glasziou PP, Irwig LM, Lijmer JG, Moher D, Rennie D, de Vet HC: Towards complete and accurate reporting of studies of diagnostic accuracy: The STARD Initiative. Ann/nternMed 2003, 138(1):40-44.

24. Bossuyt PM, Reitsma JB, Bruns DE, Gatsonis CA, Glasziou PP, Irwig LM, Moher D, Rennie D, de Vet HC, Lijmer JG: The STARD statement for reporting studies of diagnostic accuracy: explanation and elaboration. Ann/nternMed 2003, 138(1):W1-12.

25. Stroup DF, Berlin JA, Morton SC, Olkin I, Williamson GD, Rennie D, Moher D, Becker BJ, Sipe TA, Thacker SB: Meta-analysis of observational studies in epidemiology: a proposal for reporting. Meta-analysis Of Observational Studies in Epidemiology (MOOSE) group. JAMA 2000, 283(15):2008-2012. 
26. Moher D, Cook DJ, Eastwood S, Olkin I, Rennie D, Stroup DF: Improving the quality of reports of meta-analyses of randomised controlled trials: the QUOROM statement. Quality of Reporting of Meta-analyses. Lancet 1999, 354(9193):1896-1900.

27. Liberati A, Altman DG, Tetzlaff J, Mulrow C, Gotzsche PC, loannidis JP, Clarke M, Devereaux PJ, Kleijnen J, Moher D: The PRISMA statement for reporting systematic reviews and meta-analyses of studies that evaluate healthcare interventions: explanation and elaboration. BMJ 2009, 339: b2700.

28. Moher D, Liberati A, Tetzlaff J, Altman DG: Preferred reporting items for systematic reviews and meta-analyses: the PRISMA statement. BMJ 2009, 339:b2535.

29. Matsubayashi M, Kurata K, Sakai Y, Morioka T, Kato S, Mine S, Ueda S: Status of open access in the biomedical field in 2005. J Med Libr Assoc 2009, 97(1):4-11.

30. Langan S, Schmitt J, Coenraads PJ, Svensson A, von Elm E, Williams H: The reporting of observational research studies in dermatology journals: a literature-based study. Arch Dermatol 2010, 146(5):534-541.

\section{Pre-publication history}

The pre-publication history for this paper can be accessed here: http://www.biomedcentral.com/1471-2431/11/27/prepub

doi:10.1186/1471-2431-11-27

Cite this article as: Meerpohl et al:: Are pediatric Open Access journals promoting good publication practice? An analysis of author instructions. BMC Pediatrics 2011 11:27.

\section{Submit your next manuscript to BioMed Central and take full advantage of:}

- Convenient online submission

- Thorough peer review

- No space constraints or color figure charges

- Immediate publication on acceptance

- Inclusion in PubMed, CAS, Scopus and Google Scholar

- Research which is freely available for redistribution

Submit your manuscript at www.biomedcentral.com/submit 